Revue belge de géographie

1 | 2013

Modelling and benchmarking of borders

\title{
La coopération transpyrénéenne : un modèle de gouvernance de proximité ?
}

Trans-Pyrenean cooperation: A model of local governance?

\section{Adenane Djigo}

\section{(2) OpenEdition}

\section{Journals}

\section{Édition électronique}

URL : http://journals.openedition.org/belgeo/10594

DOI : 10.4000/belgeo.10594

ISSN : 2294-9135

Éditeur :

National Committee of Geography of Belgium, Société Royale Belge de Géographie

Référence électronique

Adenane Djigo, «La coopération transpyrénéenne : un modèle de gouvernance de proximité ? », Belgeo [En ligne], 1 | 2013, mis en ligne le 31 octobre 2013, consulté le 30 avril 2019. URL : http:// journals.openedition.org/belgeo/10594; DOI : 10.4000/belgeo.10594

Ce document a été généré automatiquement le 30 avril 2019.

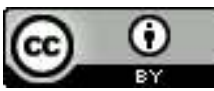

Belgeo est mis à disposition selon les termes de la licence Creative Commons Attribution 4.0 International. 


\section{La coopération transpyrénéenne : un modèle de gouvernance de proximité?}

Trans-Pyrenean cooperation: A model of local governance?

\section{Adenane Djigo}

\section{Introduction}

Comment articuler les espaces transfrontaliers alors que ceux ci se fondent sur plusieurs logiques de gouvernance (locale, régionale, étatique et européenne) parfois contradictoires? L'idée force de l'article est de montrer l'importance de la notion de proximité comme un des éléments structurant les pratiques et les discours de la coopération transfrontalière. L'analyse est centrée sur l'exemple de la partie orientale de la frontière transpyrénéenne. Les espaces d'étude sont appréhendés dans leur globalité (au delà de la ligne frontalière). Cet article est issu des travaux de recherche dans le cadre d'une thèse en cotutelle entre les Universités de Perpignan en France et de Gérone en Espagne, financée par contrat doctoral. Au delà des références bibliographiques, il s'appuie en partie sur des enquêtes de terrain, notamment des entretiens réalisés avec des acteurs locaux et régionaux entre novembre 2011 et février 2013. La coopération transfrontalière envisagée au niveau local ou de proximité est de nature variable selon qu'il s'agisse de prestation de services, de l'ébauche d'une intercommunalité transfrontalière, ou de coordinations en réseaux qui dépassent le voisinage frontalier. L'article commence par une contextualisation et des éléments méthodologiques, se poursuit par une présentation du périmètre d'étude, et présente enfin les résultats obtenus sur les types de frontières et les axes de coopération. 


\section{Objectifs et méthodologie}

2 Cette première partie traite du contexte politique et des discours sur la coopération transfrontalière sur la frontière franco-catalane. Au-delà d'un point préalable sur les représentations, sans entrer dans les détails, elle vise à préciser les principaux concepts dont il est question, notamment celui de la proximité.

\section{Contextualisation}

3 Entre le département des Pyrénées-Orientales et les provinces catalanes, les relations transfrontalières se distinguent selon deux aspects : la coopération initiée par des acteurs issus de l'agglomération de Perpignan et de la capitale de la Catalogne, et une coopération sous une autre forme entre les communes et comarcas proches de la frontière. Les premiers acteurs ont mis en place des réseaux interurbains entre pôles (Barcelone et Perpignan, d'abord) puis entre villes moyennes (Perpignan et Gérone). Leur démarche, pouvant être stratégique (Maury, 2008), car la frontière peut être utilisée comme ressource politique, se différencie de celle des acteurs situés sur les espaces très proches de la frontière car elle relève moins d'une coopération en réseau (marketing territorial, lobbying pour transports). Il s'agit plutôt de traiter un ensemble de questions qui se posent à une échelle dépassant la ligne frontalière classique, comme celles liées aux incendies ou les risques concernant le milieu littoral et aquatique à travers le tourisme et les espèces menacées. Toutefois, ces deux types de coopération semblent ne pas se confondre, même si les acteurs utilisent les mêmes canaux concernant les financements européens (et en partie leur entourage politique national). Notre étude reste néanmoins centrée sur les seconds dans la mesure où leurs territoires afférents sont frontaliers, généralement ruraux ou semi-urbanisés.

4 Si les discours liés à ces stratégies transfrontalières relèvent de représentations différentes, la réalité territoriale oblige à tenir compte d'une porosité partielle de la frontière et de l'inexistence d'un marché de l'emploi transfrontalier à l'échelle d'un Eurodistrict catalan. Certes les Etats de part et d'autre relèvent de nature différente, mais le pragmatisme de certains acteurs a amené des réflexions nouvelles et surtout d'autres façons d'appréhender la gestion transfrontalière; ainsi, au-delà des périmètres institutionnels et de la coopération thématique, la gouvernance de ces espaces est de plus en plus coordonnée à la fois à l'échelle très localisée de chaque problématique et au niveau d'une réalité transfrontalière avérée (espaces vécus au-delà de la frontière ou bassin de vie éventuel). L'Union Européenne a appuyé et facilité ces coordinations locales transfrontalières avec notamment le statut de GECT assez flexible car sui generis, en plus de ses financements: se développe une "subsidiarité transfrontalière" avec une gouvernance pouvant relever principalement du local. Entre l'Etat, ses fonctions classiques et sa reconfiguration, l'Europe et son innovation (et transfert d'innovation) en termes de soutien aux politiques publiques, les acteurs locaux construisent un territoire, vu ou imaginé sous l'angle de la complémentarité. La frontière franco-espagnole, sur sa partie orientale, connaît une forte évolution de la coopération: une mobilisation des acteurs territoriaux, de certains hommes politiques, des universités, des chambres de commerces, entre autres. Depuis INTERREG I au niveau communautaire et sa déclinaison locale, la coopération est passée de thématiques culturelles, ou d'ordre interrégional, de 
l'interconnaissance et d'études de faisabilité, à une véritable coordination de proximité. $\mathrm{Au}$ final, la notion de proximité, dans ses diverses acceptions, est-elle un référentiel pouvant contribuer à l'évaluation d'un espace ou territoire transfrontalier?

\section{Concepts et méthodologie}

5 Définie comme un processus de coordination d'acteurs [...] pour atteindre des buts propres [...] dans des environnements fragmentés, incertains par Le Galès (1995), la gouvernance relève dans un contexte transfrontalier de plusieurs niveaux de décisions pouvant être appréhendés de haut en bas ou de façon inverse. Quant à la proximité, tantôt définie comme un espace de l'interconnaissance, ou la co-présence, elle serait aussi l'échelle qui réduit l'espace social urbain à une dimension de la quotidienneté (Giband, 2010). Avec ce concept polysémique, paradigme issu en France d'un contexte urbanistique (politique de la ville), l'enjeu ici est de faire une analyse en terme de gouvernance, et à l'échelle d'espaces transfrontaliers. De ce fait, qu'apporte le concept de proximité aux projets de territoires en général et transfrontaliers en particulier?

La coopération transfrontalière envisagée ici concerne les relations catalano-catalanes, dans un contexte de deterritorialisation / reterritorialisation (Brenner, 2010), et de gouvernance structurée de plus en plus entre les échelons local et européen. Notre analyse se nourrit d'éléments bibliographiques et surtout des divers discours récoltés lors de nos entretiens avec les acteurs locaux, notamment des élus mais aussi des techniciens (urbanistes par exemple) ou des représentants d'établissements publics locaux, sur cette partie de la frontière franco-espagnole. Notre problématique reste centrée sur la modélisation d'une frontière, déclinée ici sur les formes de gouvernance et les dynamiques de proximité construites de part et d'autre. Le territoire étant en partie construit, nous partons d'une hypothèse principale selon laquelle des périmètres de coopération institutionnelle comme l'Eurodistrict Catalan Transfrontalier (ECT) relèvent d'avantage de représentations que d'une intégration fonctionnelle. La proximité serait d'abord une problématique de vie quotidienne ou l'enjeu des politiques publiques au niveau local. Egalement, l'entité cerdane ou les Albères sont des micro-espaces intégrés, fonctionnels pour le premier et seulement topographique pour le second. Le plateau cerdan par exemple abrite la plupart des migrations alternantes enregistrées ou comptabilisées au niveau de l'espace catalan transfrontalier ${ }^{1}$. La plupart des études menées jusqu'ici ont utilisé des méthodes différentes pour une évaluation des espaces transfrontaliers.

7 Que ça soit de façon thématique ou globale, elles ont souvent procédé par une évaluation sur la base des chiffres existants (distincts de part et d'autre de la ligne frontalière) avant d'effectuer des moyennes. Même les observatoires transfrontaliers créés notamment dans le cadre de projets européens se confrontent aux mêmes contraintes. Le constat est le même pour la cartographie. Au su de ces limites, nous procédons pour les données quantitatives à des combinaisons entre des sources différentes. C'est la raison pour laquelle nous privilégions les types de coordinations, bref la gouvernance de ces espaces à travers une analyse de la logique des acteurs. Sur les aspects géopolitiques de la frontière choisie, la méthode employée est une série d'indicateurs, en l'occurrence le nombre de structures transfrontalières, et bien évidemment les représentations au niveau local et régional dans une perspective comparative. 


\section{Présentation du périmètre d'étude : les projets de coopération depuis 1980}

Dans ce contexte, le périmètre de coopération de proximité au niveau de la frontière franco-catalane serait les espaces contigus de part et d'autre (proximité géographique) et avec une certaine cohérence territoriale. Certes, en termes institutionnel ou de réseaux de coopération, cette frontière a vu l'apparition de l'Eurodistrict Catalan, qui doit illustrer ce passage de l'interconnaissance, à la gestion commune de problématiques plus localisées et partagées, dites de proximité. Notre périmètre d'étude est la bande transfrontalière au sein de cet espace, correspondant notamment au plateau cerdan et au Conflent (zone 2) et aux Albères en plus du Vallespir (zone 1) à l'est. Quant à la gouvernance et sa mise en œuvre par les acteurs locaux, elle sera l'objet des sous-parties suivantes et de la conclusion. Le découpage du périmètre en deux zones d'étude reprend une nomenclature ou classification selon des territoires historiques au Nord et les comarques au Sud. Le rajout du Conflent à la zone 2 répond à la même logique.

\section{Géographie}

9 Ce choix de périmètres est motivé par une certaine unité géographique de ces espaces et une différenciation aussi par rapport au reste constitué par la plaine du Roussillon dans le département des Pyrénées-Orientales et le sud de la province de Gérone. Selon J.F. CastexEy, les territoires de cet espace présentent deux visages qui, à première vue, semblent s'opposer. En effet, de part et d'autre de la frontière, les territoires sont structurés grosso modo de la façon suivante: un réseau de villes (ou une ville polarisante) opposé à un maillage de villages et petites villes. Cette différenciation géographique plus ou moins marquée trouve son origine dans une répartition de fonctions territoriales divergentes [...]: les fonctions urbaines et périurbaines localisées à l'est, et les fonctions rurales ou rurbaines à l'ouest. De surcroît, sur la bande littorale frontalière, le massif des Albères nuance cette configuration car il constitue un espace rural, et une sorte d'arrière-pays des stations balnéaires de la costa Brava et de la côte Vermeille. 
Figure 1. Localisation des deux périmètres d'étude.

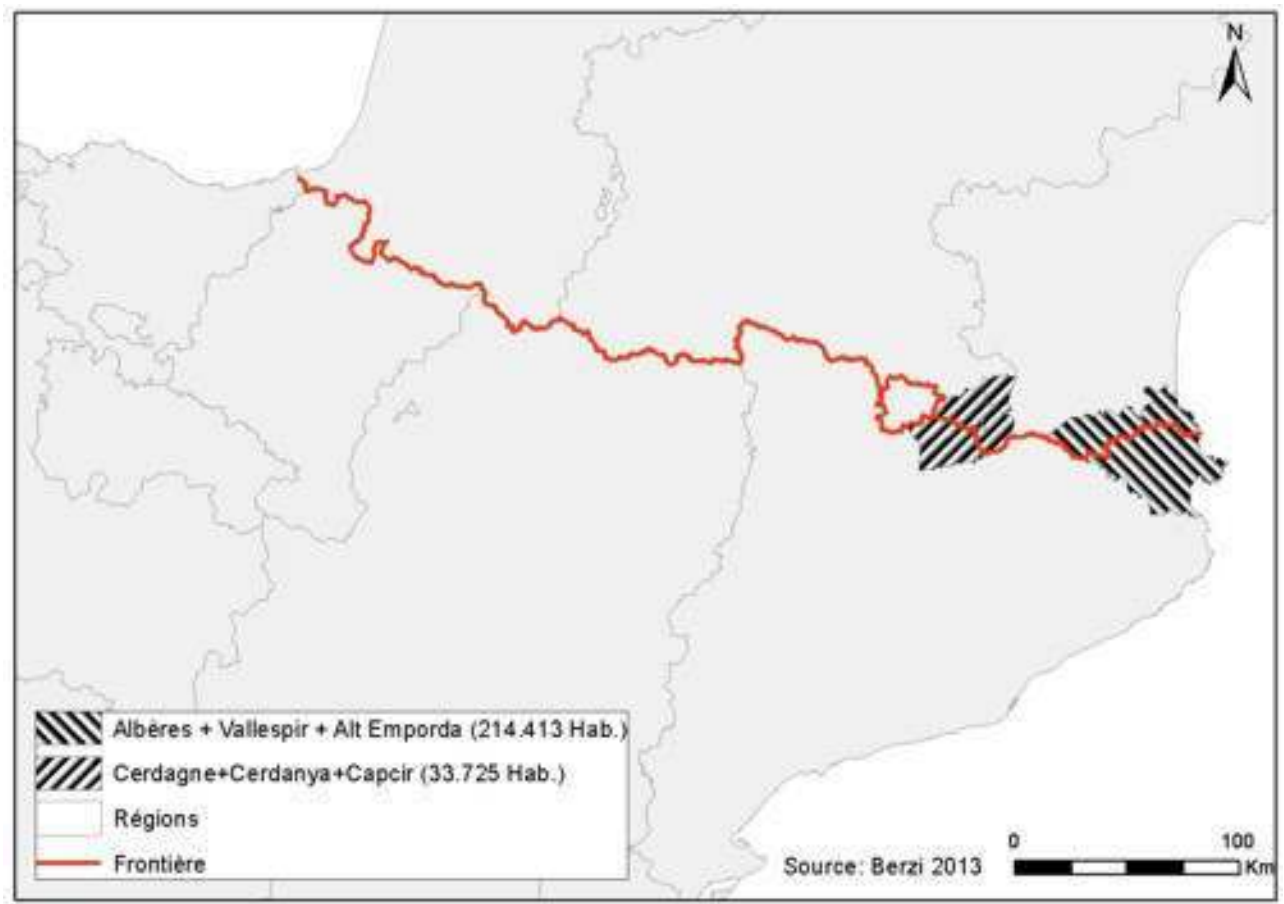

\section{Bilan diachronique de la coopération sur les deux espaces}

Si l'on fait le choix de partir du début des années 1980, marqué par la fin de la dictature espagnole et la première Convention cadre européenne sur la coopération transfrontalière, nous pouvons effectuer un bilan synthétique des types de coordination menés dans le cadre de projets locaux et surtout de mise en place d'accords et de financements. L'accent est mis ici sur quelques projets menés à l'échelon très localisé de la frontière. Il s'agit notamment d'actions symboliques comme des jumelages ou de petits projets d'infrastructures entre communes frontalières. Ainsi, dès l'année 1985 par exemple, une première phase d'interconnaissance entre entreprises et collectivités territoriales de part et d'autre de la frontière s'est traduite par quelques prospections en Espagne des chambres consulaires (Antennes de la Chambre de Commerce et d'Industrie et de la Chambre d'Agriculture en Cerdagne). Sur nos deux espaces choisis, deux binômes de communes illustrent les dynamiques sectorielles qui leur sont propres dans ce vaste espace, tantôt dit euro régional, tantôt appelé Eurodistrict : il s'agit de Bourg-Madame / Puigcerdá et Cerbère / Port-Bou. En 1989 déjà, les municipalités de Bourg-Madame et de Puigcerdá, associées à la Chambre de Commerce et d'Industrie (CCI) et au Conseil Général des Pyrénées-Orientales, forment un syndicat mixte pour procéder à une acquisition de terrains dans le cadre de l'aménagement d'un projet de zone industrielle à Puigcerdá. En 1991, sur le même plateau cerdan, Estabar et Llivia signent une charte de collaboration transfrontalière établissant un Conseil municipal commun. Le binôme Bourg-Madame Puigcerdá prend la même initiative en 1995 pour la création d'une commission mixte pour l'étude et la réalisation de projets d'intérêts communs. En 1996, de façon plutôt symbolique, les deux municipalités organisent le $5^{\text {ème }}$ Forum mondial des jeunes architectes, qui se traduira quelques années plus tard par l'aménagement concerté d'une rue commune aux deux villes : il s'agit de la concrétisation du projet intitulé Matrimoni 
Sense Frontera en 2005. Au niveau des Albères et du Vallespir, au-delà de plusieurs associations créées généralement pour la défense de l'environnement dans un cadre transfrontalier, à l'instar de Albera Viva, association double avec un statut juridique dans chaque pays, s'y est illustrée une autre dynamique: celle de la mobilisation ou de la coopération de résistance, contre un projet de ligne de Très Haute Tension, ou encore pour défendre une infrastructure existante face à de nouveaux projets d'équipements structurants. Dans la même mouvance, les mairies de Cerbère et Port-Bou se sont mobilisées récemment, en partenariat avec des usagers, pour une journée de revendication pour le maintien de la ligne conventionnelle de train (Trains Express Régionaux français et trains régionaux espagnols), qui serait menacée par l'arrivée de la Ligne à Grande Vitesse entre Barcelone et Perpignan.

11 Entre Bourg-Madame et Puigcerdá, il n'y a certes pas de jumelage ; toutefois, nous pouvons retenir, comme actions clés dans le cadre de dynamiques de coopération locale, la réalisation d'une station d'épuration en 1995, financée non pas par l'Union Européenne, mais par la Généralité de Catalogne. Au-delà, que ce soit en Cerdagne ou au niveau des Albères, les dynamiques de coopération initiées de façon très localisée au niveau de la frontière restent plutôt modestes et tantôt symboliques, comme la Diada de Cerdanya / Journée de Cerdagne, ou la Diada de l'Arbre / Journée de l'Arbre. Sur la partie orientale, en plus de Cerbère/Port-Bou, on pourrait analyser aussi les relations entre Le Perthus et La Jonquera, qui en plus de partager le massif des Albères, constituent une zone commerciale configurée selon l'effet frontière classique. Ce binôme est qualifié par un maire voisin de plus grand bureau de tabac d'Europe. Selon Mr Picas, maire du Perthus, il y a un double partage du massif des Albères entre France et Espagne. La différence de développement est due à cette différence de fiscalité, de règlementation. Le mouvement s'est plusieurs fois inversé. L'harmonisation par l'Europe, ce serait sur le long terme. Les projets en commun, on les fera malgré les différences (production d'eau, distribution). La différence n'est d'ailleurs pas un obstacle. Il se dégage déjà des thématiques de coopération propres à chacun de ces deux espaces ; c'est que nous allons tenter de démontrer dans nos résultats à partir de la typologie des frontières et de la gouvernance qui en émerge. De ce fait, audelà des structures nationales et avant de tirer avantage de la politique de cohésion européenne, des acteurs locaux, en partie des personnalités à l'instar de Henri Sicre, ancien député socialiste pour le Vallespir, Jean Castex, actuel maire de Prades et F. Calvet pour la Cerdagne ou encore Joan Armangué, ancien maire de Figuères pour l'Empordà ont su mener des coordinations transfrontalières, avec le mérite d'avoir jeté les bases de certaines thématiques de coopération; car comme ces derniers le rappellent souvent, la coopération est une question de volontarisme et une affaire de personnes. Certes, il y a la combinaison de plusieurs avancées administratives et institutionnelles au niveau des Etats comme le Traité de Bayonne par exemple, cependant, les actions qui étaient d'ores et déjà menées à l'échelle de ces micro-espaces transfrontaliers démontrent l'existence de dynamiques de proximité ou de voisinage sectoriels bien avant la facilitation européenne.

\section{Une coopération européenne au bénéfice des projets locaux ?}

12 A ce titre, simultanément aux dynamiques propres à nos deux espaces transfrontaliers, l'acteur européen a entendu encourager dès le début des années 1980 les espaces frontaliers à travailler ensemble. Son apport a été essentiel en termes de cofinancement de projets, de transfert de valeurs et de positionnement aussi : l'Union Européenne ayant 
d'abord favorisé les acteurs régionaux, en a fait un échelon important dans une vision communautaire de l'aménagement du territoire. Mais aussi, elle a su être le nouvel échelon global ou supranational combiné à celui local des territoires ou espaces frontaliers, avec l'Etat en tant qu'acteur intermédiaire. A propos de l'évolution de la coopération dans le cadre communautaire, nous nous recentrons sur les apports occasionnés selon les thématiques de coopération qui ressortent de nos deux espaces d'étude : à savoir la protection de l'environnement au niveau des Albères et du Vallespir et la montée en puissance de la thématique de la santé en Cerdagne, ainsi que d'autres problématiques annexes ou similaires.

13 Ainsi, en matière de gestion de l'environnement, il y a certes eu en Catalogne une Charte au début des années 1990, ou plutôt la Declaració de Reserva de Biosfera de l'Empordà (Armangué, 2010), qui devait être planifiée au niveau de l'UNESCO et étendue à l'autre bande de la frontière. Toutefois, cette Charte ne concerne que les comarcas catalanes de l' Atl-Empordà et du Baix-Empordà. Au-delà, en septembre 1996, la Compagnie Nationale du Bas-Rhône Languedoc et son homologue catalan des Aigues Ter Llobregat créent un Groupement Européen d'Intérêt Economique (GEIE) pour le projet d'aqueduc LanguedocRoussillon-Catalogne; ce projet d'initiative catalane d'adduction d'eau depuis le Rhône, malgré un fort enjeu géopolitique (notamment entre Aragon et Catalogne), restera à l'étude et les scénarios de réalisation n'iront pas plus loin. Une Directive cadre sur l'eau encourage depuis 2000 la coopération entre Etats. En Espagne, l'eau, symbole de la solidarité nationale et interterritoriale, est un des rares éléments relevant principalement du pouvoir central, même si les Communautés Autonomes peuvent y participer. C'est le Traité d'Amsterdam qui a introduit la notion de développement durable dans la stratégie communautaire. L'innovation et le projet deviennent ainsi la base de l'action : ce qui est promu et confirmé par la Stratégie Europe 2020, avec son volet environnemental. D'ailleurs, une déclinaison de ces objectifs européens, établis dans un contexte de crise financière internationale, existe au niveau de la Catalogne par exemple avec une Estratègia Catalunya 2020. Ce paradigme du développement durable influence les choix effectués à propos des équipements. L'exemple de la mobilisation contre la ligne à Très Haute Tension restera symbolique. Aussi, le choix d'enterrer cette ligne est-il $a$ posteriori environnemental, et certainement un compromis entre revendications locales de toutes sortes et projets structurants. Malgré le potentiel d'articulation et de planification des espaces dans le domaine de l'environnement, surtout au niveau des Albères, il y a moins de projets aboutis que de mobilisations contre certains d'entre eux. A l'échelle des Pyrénées, des initiatives comme le GEIE FORESPIR, regroupant dans une même structure les autorités en charge de la gestion des forêts et des propriétaires de part et d'autre, sont cependant quelques rares exemples de coordination dans ce domaine. Aujourd'hui, l'une des dernières "innovations" est le projet de création d'un parc naturel marin transfrontalier; l'initiative ne date pas d'hier mais restera peut être un "projet" tout court, vu les nombreuses limites (contraintes juridiques, dissymétries administratives, et stratégies politiques différentes); et malgré une ébauche de mise en œuvre d'un programme sur la biodiversité marine (MIRO) sur le même espace de projet. Même si, ailleurs en Europe, des projets similaires semblent réussir comme celui du parc marin transfrontalier des Bouches de Bonifacio (sur la base d'un accord de coopération toutefois, sans personnalité juridique), au niveau de la frontière franco-catalane, il semble que les acteurs locaux soient plus intéressés par des initiatives relevant de la patrimonialisation ou du tourisme. L'illustration est le projet en cours de voie verte 
transfrontalière dénommé Enllaç. Pendant que certaines communes des PyrénéesOrientales tentent de mettre en œuvre des Agenda21 transfrontaliers (Sorède, AmélieLes-Bains), la planification, en matière d'urbanisme notamment, demeure territorialisée selon des périmètres administratifs relevant de chaque Etat, n'intégrant par conséquent pas ou très peu la donne transfrontalière.

Sur le plan sanitaire, la Catalogne se caractérise par la séparation entre financement et fourniture de soins, et dispose ainsi d'un organisme, CatSalut, créé en 1990 et chargé de la planification de l'offre hospitalière du secteur public. En France, les secteurs sanitaires et schémas régionaux d'organisation sanitaire (SROS) sont instaurés en 1991. Ce fut la première étape en matière de planification de l'offre hospitalière dans les cadres régionaux. Ce qui se confirme avec les "Ordonnances Juppé" de 1996. En 2001, un accord est signé entre l'Agence Régionale de l'Hospitalisation (ARH) du Languedoc-Roussillon, le centre hospitalier de Perpignan et l'Hôpital de Puigcerdá pour la prise en charge des urgences chirurgico-médicales et des accouchements des patients de Cerdagne française par l'Hôpital voisin. En 2003, la Généralité de Catalogne, la région Languedoc-Roussillon, l'ARH et CatSalut signent le protocole d'accord initiant le lancement d'une "étude de faisabilité de la création d'un hôpital commun" transfrontalier dans le cadre de l'Eurorégion et d'un appel à projet INTERREG III A. Depuis mars 2004, les SROS doivent, d'après une circulaire, articuler les moyens des établissements de santé avec la médecine de ville et avec les secteurs sociaux et médico-sociaux, tout en tenant en compte pour les bassins de vie concernés de l'offre de soins des régions limitrophes et des territoires frontaliers. Quelques mois plus tard, une réunion est organisée entre l'ARH, CatSalut, La Fondation "Hôpital de Puigcerdá" pour lancer des études portant spécifiquement sur les aspects juridiques et financiers du projet d'hôpital transfrontalier. Depuis lors, la conception du projet d'hôpital a démarré (octobre 2005). En 2006, les ministères catalans et français de la santé signent une déclaration conjointe d'intention sur la coopération dans le domaine de l'accès aux soins de proximité pour les patients de Cerdagne et de Capcir. Toutefois, les autorités françaises refuseront que le Traité de Bayonne ne serve de cadre de référence à l'établissement du consortium inter-administratif chargé de gérer l'hôpital de Puigcerdá car le secteur de la santé reste de la responsabilité des Etats membres de l'Union Européenne. De ce fait, une fondation privée de droit catalan a été créée dans l'attente d'un accord intergouvernemental qui paraissait être la seule solution envisageable pour pérenniser le projet en levant toute insécurité juridique.

Après que le futur HCTC (Hôpital Commun Transfrontalier de Cerdagne) se soit doté d'un Conseil d'Administration, en décembre 2010, une première pierre est posée à Ur pour un futur abattoir : ce projet est en lien avec l'HCTC, à l'instar du spa en cours de réalisation à Llivia ou encore un golf à l'étude sur cette même enclave espagnole. A terme, l'Ensemble cerdan, comme dirait François Mancébo, devrait être un bassin de vie, structuré au moins sur le plan sanitaire, et coordonné avec Perpignan et la Catalogne. A l'issue d'un conseil des ministres en France datant du 9 janvier 2013, Laurent Fabius a présenté un projet de loi autorisant la ratification de l'accord-cadre entre la République française et le Royaume d'Espagne sur la coopération sanitaire : cet accord, signé à Saragosse en 2008, ainsi que son accord d'application signé à Angers, visent notamment une simplification des démarches administratives et financières; ce nouveau cadre juridique devra permettre d'offrir une complémentarité des soins, en évitant la duplication des moyens disponibles en France et en Espagne. 


\section{Types de frontières, axes de coopération et gouvernance afférente}

Compte tenu de la diversité dans la définition de la proximité, le concept peut être employé au pluriel: les proximités dans leurs divers aspects seront illustrées dans la sous-partie suivante sur la base de la perception des acteurs interrogés. Une lecture pourra y être faite à propos des motivations qui permettent d'expliquer le caractère plus ou moins perméable de la frontière et la proximité des acteurs décisionnels français et espagnols. Nous reviendrons sur une typologie des proximités selon qu'elles soient géographique ou topographique, culturelle, ou encore institutionnelle ou organisationnelle (tableau 1). Parallèlement aux proximités, même si certains acteurs parlent généralement d'une frontière artificielle, un retour sur le concept de frontière s'impose. Selon M.C Fourny (2005), si la frontière a produit le territoire et a défini des territoires frontaliers, ces territoires reproduisent de la frontière, refondent la limite qui en fait l'existence. La frontière est donc moins remise en cause qu'affectée d'un nouveau statut, d'objet signifiant des différentiations et des identités, mais générant dans le même temps son propre dépassement (Fourny, 2003).

\section{Typologie des "proximités" et des frontières}

En terme de relief, les espaces nous concernant relèvent d'une vallée pour la Cerdagne et d'un massif à l'est, en l'occurrence celui des Albères. Sur la géographie physique de la région de Cerdagne, Séchet et Keerle (2010) l'ont décrite comme constituée d'une certaine unité morphologique, assez nettement délimitée par plusieurs massifs et sommets (Carlit, Puigmal, Puigpedros...) ; le plateau cerdan, qui correspond à la haute vallée du Sègre, est surplombé dans sa partie nord-est par le Capcir. La Cerdagne n'a longtemps été accessible que par des cols régulièrement enneigés: col du Puymorens vers la haute Ariège et Toulouse, col de la Perche vers Perpignan, défilé du Baridà vers La Seu d'Urgell puis Lleida. La rutlla ou ligne frontalière y est aujourd'hui quasiment invisible sur une plaine semi-urbanisée. Au niveau est, la frontière est plutôt montagneuse et boisée, et surtout rurale. Ainsi, a priori, au niveau des deux zones, la proximité est avant tout géographique ; selon leur système d'organisation, la proximité dite institutionnelle se structure à des degrés similaires. 
Figure 2. Le relief au niveau de nos zones d'étude.
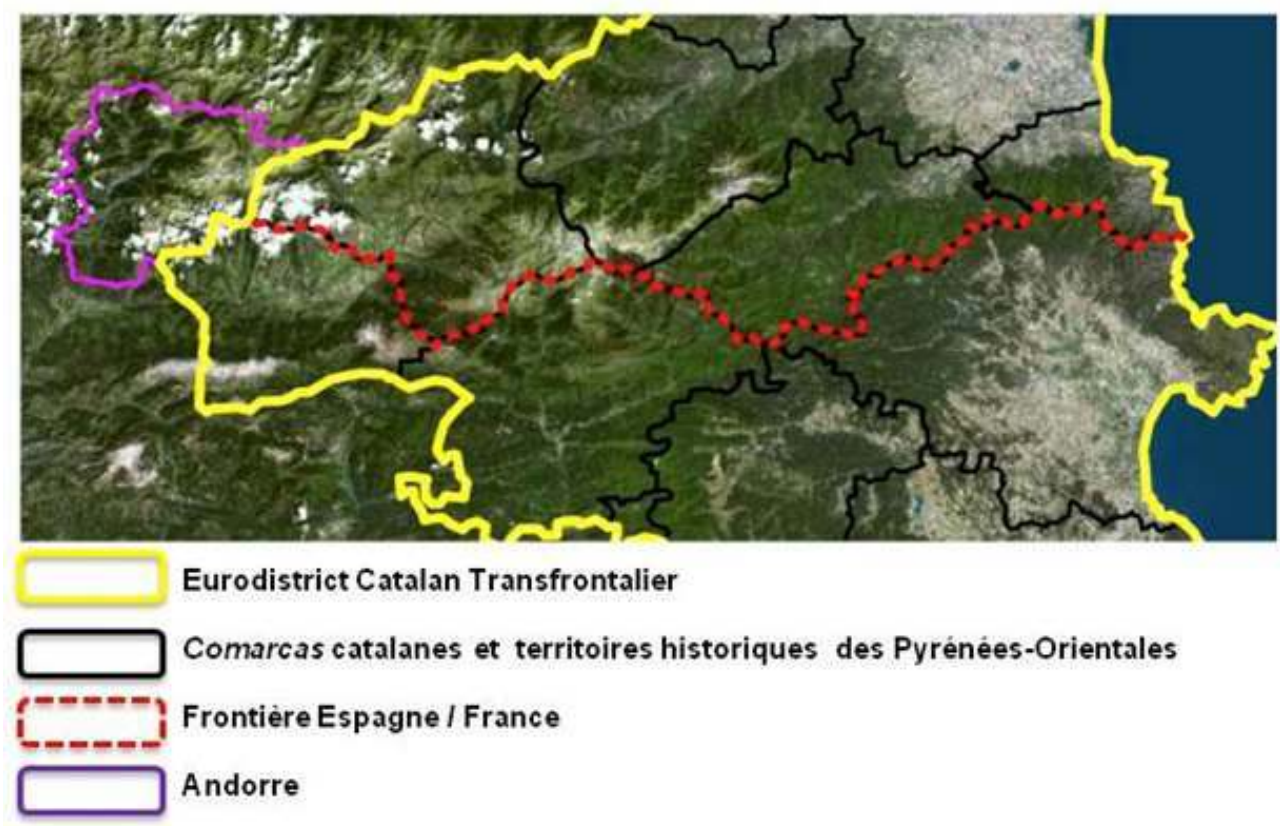

Source : Google Maps résonante. La proximité c'est un concept. Qu'est ce qu'on peut faire ensemble qui soit de proximité (spatiale ou linguistique)? Certaines actions ne sont pas concevables dans certains périmètres (100 $\mathrm{km}$ ) selon la députée européenne Marie-Thérèse Sanchez-Schmidt. Selon le maire de Saillagouse, la proximité, c'est l'inverse de ce que fait l'Etat (fermeture des services). C'est donc le fait que les gens trouvent le service devant eux, ou même avec la nouvelle technologie une réponse physique à leurs besoins. Ça fait partie des moteurs de la coopération transfrontalière. Le géographe Jean Castex-Eyaffirme lors d'un entretien que certains diront que l'Eurodistrict, ce n'est déjà plus de la proximité car trop vaste ; c'est donc un assemblage de proximités. La proximité est un préalable pour que la coopération soit effective. A l'interrégional, c'est plutôt du lobbying à Bruxelles pour les grandes infrastructures. La proximité, c'est une construction européenne à l'échelle locale. La proximité nécessite forcément une relation directe, une continuité; le réseau peut relever de la proximité aussi avec de bons moyens de transports (Exemple du Comité Eurozone entre Malmö et Copenhague, il ya de la proximité avec le pont les reliant). Ici, pour le moment, la liaison n'est pas bonne. Un chargé de l'aménagement du territoire à la Préfecture des Pyrénées-Orientales définit la proximité comme des actions de coopération qui puissent bénéficier aux habitants de la zone, par exemple, mettre en place une ligne de bus entre Perpignan et Gérone, ou encore, dans les domaines de la culture et sanitaire, la lutte contre les incendies, le courrier aussi et surtout l'emploi.

Un chargé de mission du Conseil Général affirme, quant à lui, que sur la proximité, les enjeux sont liés au territoire dont on parle, et difficilement transposables et détachables $\mathrm{du}$ territoire: ce sont des choses concrètes et opérationnelles comme les itinéraires communs; plus on s'éloigne du territoire, moins c'est concret, donc c'est une approche différente. La proximité, dans sa conception des années 90 , c'est la connaissance mutuelle et c'est le premier enjeu rappelle Estève Valls, un technicien de la Communauté d'agglomération 
Perpignan Méditerranée. Un politique, représentant du parti CDC (Convergencia Demòcratica de Catalunya) dans les Pyrénées-Orientales, souligne que la coopération transfrontalière évoque déjà la proximité, la capacité de décider soi-même, la responsabilité; pouvoir travailler ensemble, mettre en place sur un territoire des décisions utiles, rompre plusieurs siècles d'autarcie car on s'était tournés le dos. Il rajoute que la proximité c'est être capable de gérer sa vie sur un petit territoire : subsidiarité. $L a$ proximité doit donc être gérée dans la proximité : c'est la démocratie et c'est un principe européen. Le mot de proximité, d'après le responsable du pôle Europe de la région LanguedocRoussillon, revient sur le discours de Jean Claude Gayssot (Vice-Président de la Région LanguedocRoussillon en charge des relations européennes, ancien ministre des transports). Pour lui, il n'y a pas d'Europe sans les citoyens. La politique de cohésion est peut être celle qui exprime le plus la proximité et la solidarité de l'Europe des territoires. Il y a une stratégie ascendante partant des territoires.

\section{Thématiques de coopération}

L'eau par exemple est un thème potentiel de coopération que ce soit en Cerdagne ou dans les Albères (souvent considéré comme moyen de régulation de conflits locaux) ; d'ailleurs, des projets portèrent sur la rivière Sègre au niveau de la Cerdagne pendant la programmation INTERREG III; d'autres thématiques, comme le déneigement, sont concrètes, et pourraient être l'objet de partenariat. Dans le cadre de notre raisonnement, le choix a été fait d'illustrer l'article par deux thèmes récurrents et spécifiques à chaque espace, et surtout avec des projets en cours.

21 En France et en Espagne, la santé ou la lutte contre les incendies ne sont pas des compétences municipales. L'hôpital transfrontalier de Cerdagne, au-delà de ses aspects administratifs et techniques, a démontré le choix et la montée en puissance de la coopération et de la planification sanitaire notamment, du fait d'un contexte (présence historique de certains types d'établissements sanitaires) et de besoins locaux (surtout saisonniers dus au tourisme) propres. L'attractivité touristique de la région a un impact sensible sur les besoins de soins, notamment en matière de traumatologie. L'argument démographique a été pour défendre un projet présenté comme un moyen d'améliorer la réponse aux besoins de soins hospitaliers des populations et comme une possibilité d'assurer la pérennité des établissements du plateau cerdan, et de sauvegarder les emplois (Séchet et Keerle, 2010). 


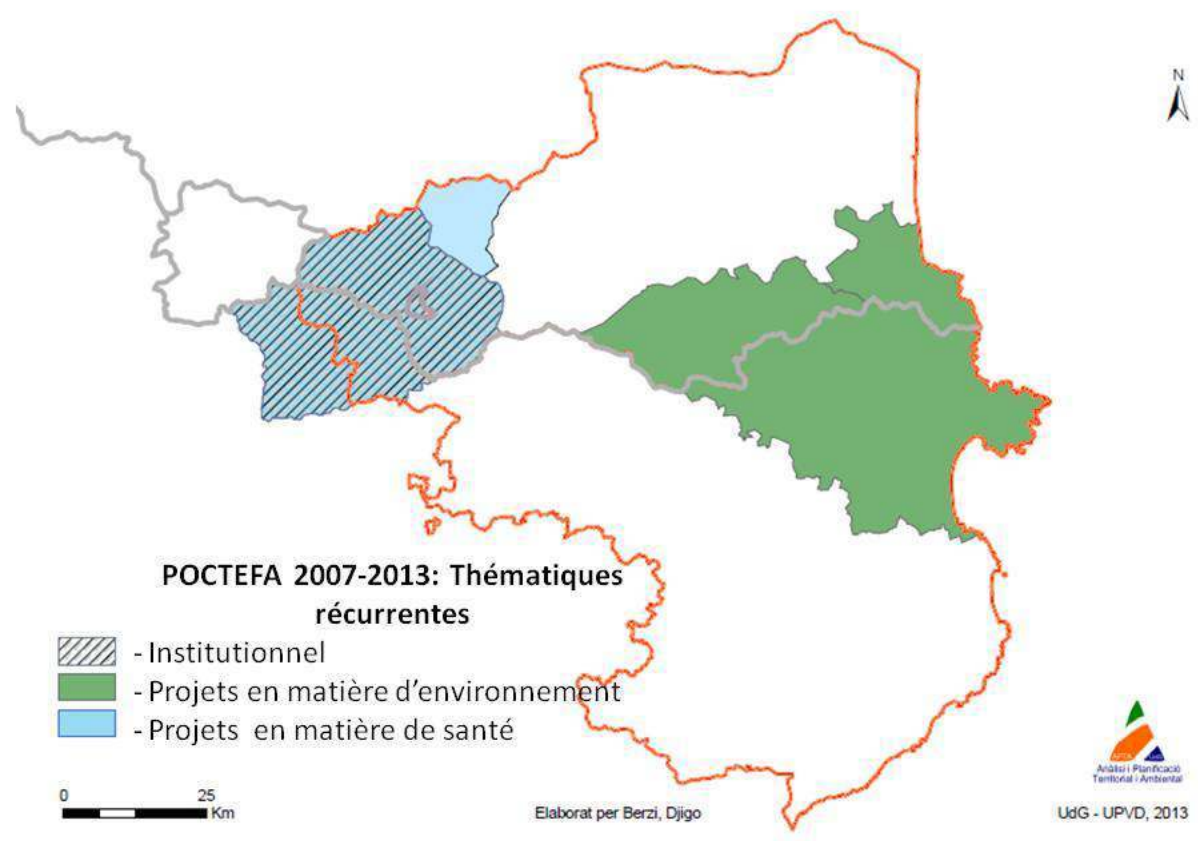

Pour la préservation de l'environnement, nous illustrons cette thématique avec la lutte contre les incendies dans les Albères. PRINCALB était destiné à l'élaboration d'un plan transfrontalier de protection incendie et la mise en place d'équipements transfrontaliers. Il fut commencé en 2009, ayant pris fin en 2011, avec un coût global de 1213000 euros, dont 149625 euros du Conseil Général des Pyrénées-Orientales, 80000 euros de financeurs divers, 247000 euros de la Generalitat de Catalunya et 736875 euros de fonds FEDER (Payrot, 2011) En effet, dans son étude sur la protection du massif, M. Payrot rappelle que si nous pouvons considérer pour acquis que le massif de l'Albera est un massif particulier et remarquable, autant par sa situation géographique que par son intérêt reconnu au niveau naturel et paysagiste, il est malheureusement aussi très exposé aux incendies. C'est même un des massifs qui a le plus brûlé ces cinquante dernières années, avec presque $93 \%$ de sa superficie affectée. Le défi demeure la coordination entre autorités locales françaises et catalanes. Et l'on peut se demander ce qui reste des projets de coopération au-delà de la durée des programmes. Il existe des coopérations sur des opérations, mais à la demande seulement; ce n'est pas automatique et c'est au cas par cas d'après le maire de la commune française de Maureillas, qui rajoute : une coopération serait utile car le feu n'a pas de frontière; ça fait quatre ans qu'une convention d'intervention réciproque est en attente de signature. Tout de même, des méthodes d'évaluation et d'intervention communes ont pu émerger du projet PRINCALB. Quant au projet de parc marin transfrontalier, il demeure, en France, une démarche nationale avec un périmètre sur la côte Vermeille. 
Figure 4. Périmètre d'étude avec quatre projets, en cours ou terminés.

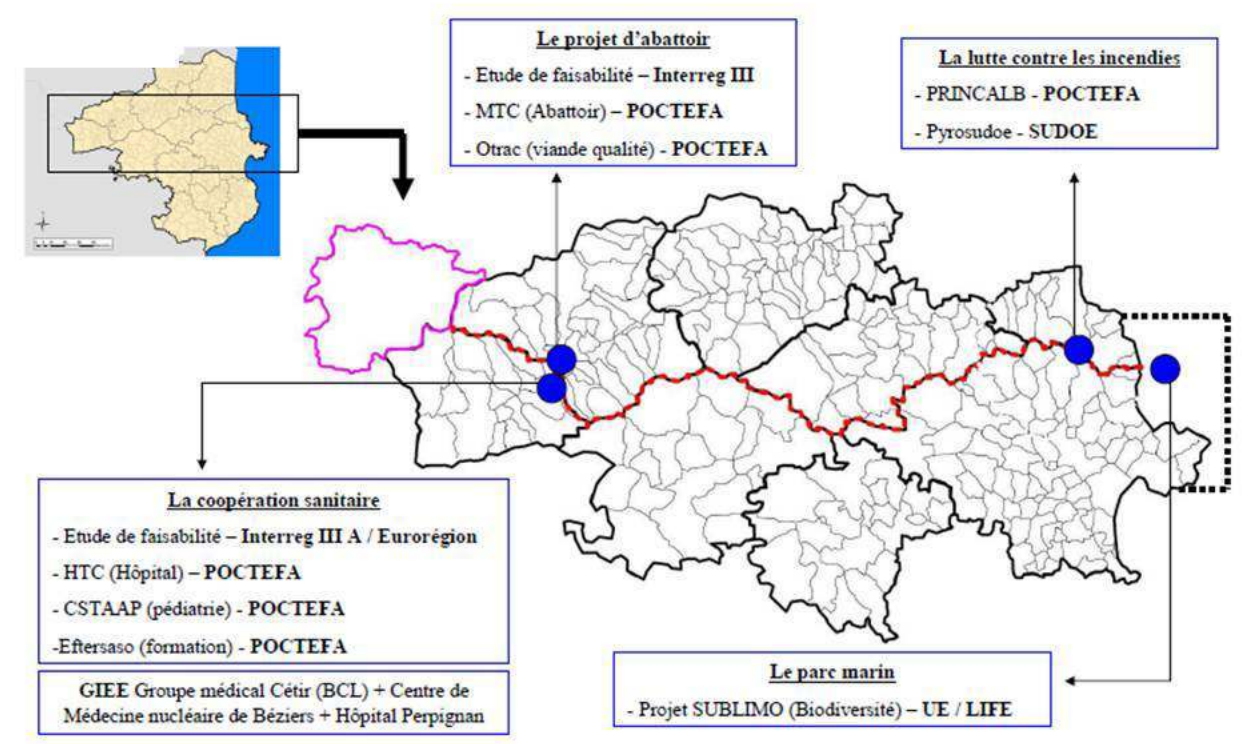

\section{Gouvernance transfrontalière, stratégies et représentations locales}

L'Entité cerdane est constituée par la présence de divers acteurs: le Consorcio BourgMadame/Puigcerdá, la Fondation Hôpital Privé, le GECT de Hôpital, la CCI et autres chambres consulaires, le Pays terres romanes, le GECT Pyrénées Cerdagne (qui inclut le Consell Comarcal au Sud et la Communauté de communes côté Nord), l'Associacio de municipis, l'Association Estavar/Llivia et d'autres syndicats mixtes. Xavier OliverasGonzalèz a répertorié les "entités de coopération locale" au niveau de l'ensemble cerdan au nombre de 35: quatre côté sud et 31 côté français (dont des Syndicats Intercommunaux à Vocation Unique ou Multiple)! Sur la Cerdagne française, il existe déjà un Parc Naturel Régional et un Pays ; l'enjeu ici aussi serait sa coordination avec la structure côté sud. Or, au niveau des Albères, la gestion de l'environnement est un enjeu réel, une problématique partagée mais le seul projet de Parc Naturel Régional est resté une initiative de développement local "Albères Pyrénées-Méditerranée". Les actions menées jusque-là l'ont été dans un cadre associatif : avec notamment "Albera Viva" au nord, "Albera Viva" au Sud; et "Cap de Creus Saint Vicenç" sur le territoire des communes littorales de part et d'autre de la frontière. Enfin, ce territoire fait partie du Réseau européen des territoires du liège (RETECORK), qui dépasse toutefois le cadre local car couvrant également le Portugal et l'Italie.

Sur la scène des relations transpyrénéennes, entre Catalogne et France (notamment Languedoc-Roussillon et Pyrénées-Orientales), il y a beaucoup d'acteurs au niveau local, en plus des interactions entre niveaux de pouvoir et de décisions jusqu'à l'échelon européen : les notables locaux et leur jeu sur l'échiquier européen, les représentations locales; des conflits politiques aussi (différences entre Conseil Général et ville de 
Perpignan et/ou Perpignan Méditerranée Communauté d'Agglomération). Dans les Pyrénées-Orientales, le gros paradoxe urbain du département est que la ville de Perpignan représente un quart de la population. Au delà de ce pôle départemental, les villes les plus grandes en population sont Canet, Saint-Estève, Toulouges autour de 10000 habitants chacune; et elles sont toutes localisées autour de Perpignan, avec un rapport de 1 à 10. Du fait de cette réalité de géographie humaine, précise une responsable de la coopération du niveau de l'agglomération, il y a une grande méfiance du département visà-vis de Perpignan, qui concentre tout (loisirs, hôpitaux) et toujours la peur d'être mangé par cette ville. Pourtant, les autres villes croissent grâce à Perpignan et se méfient de peur d'être absorbées (donc, elles grandissent contre). Le Conseil Général, quel que soit la couleur politique, a toujours pratiqué dans ses choix la contradiction avec la ville de Perpignan : il se démarquait pour exister et représentait les autres populations (ruralité). En 1986, lors de la signature de la Convention pour la première rencontre de la future Eurorégion, sur tout ce qui était aménagement, on notait une réticence interne au département. Le Conseil Général a été présidé par Guy Malé (décédé en cours de mandat), René Marquez puis C. Bourquin. Par exemple, Malé et Alduy étaient des élus de la droite française! En 1993, les choses se crispent au niveau de la coopération transfrontalière : J.P Alduy mise sur le Sud alors que Bourquin s'y oppose ; ainsi, les différences d'orientations entre ces deux personnalités, voire entre les tendances politiques locales (droite et gauche), deviennent systématiques sur tous les plans. J.P Alduy faisait des relations avec le Sud un élément de sa conception de développement du département car son atout était d'avoir des liens de l'autre côté. En tant qu'opposant à Alduy, Bourquin s'oppose au leitmotiv de la coopération avec Barcelone.

Le concept "transfrontalier", lui-même serait une représentation en réponse à la périphérisation nationale et régionale: celle nationale pour les Pyrénées-Orientales et régionale pour la province de Gérone par rapport l'agglomération barcelonaise. Après l'“Archipel roussillonnais" et "Catalogne Nord" comme conceptions ou représentations au niveau des Pyrénées-Orientales, le passage de la coopération régionale à celle de proximité s'est illustrée par une mobilisation des "petits acteurs" pour l'Eurodistrict ou d'autres GECT ; étant donné cette volonté européenne de mettre de nouveaux acteurs dans cette complexification des politiques publiques, les périmètres de coopération deviennent l'occasion de défendre certains paradigmes ou tout simplement des opportunités financières. La frontière franco-catalane est une zone marquée d'abord par des relations anciennes de voisinage, souvent régulées par des accords (Lies et passeries, Traité de Barcelone), ensuite, par une histoire commune et notamment une langue partagée. Il s'agit d'une frontière ayant connu une trajectoire spécifique même si certaines caractéristiques et dynamiques restent communes à la frontière pyrénéenne dans son ensemble voire à d'autres frontières européennes. Situées en aval de ce jeu à plusieurs niveaux entre acteurs, les autorités locales l'infléchissent par des stratégies qui leur sont propres, et, peut-être un peu plus surprenant, contribuent à la définition de nouveaux procédés, donc aux transferts de normes “institutionnelles", pas au même titre que l'Union Européenne cependant. Au niveau de la région autonome catalane et dans le département des Pyrénées-Orientales, nous estimons qu'il y a eu diverses manières de se saisir des opportunités offertes par la politique régionale communautaire. Si les autorités catalanes ont développé une certaine culture des projets transfrontaliers et de positionnement au niveau des institutions de Bruxelles, avec un projet cohérent et renouvelé, une continuité malgré des divergences internes, les Pyrénées-Orientales sont marquées par l'absence d'un acteur fort à l'instar de la Generalitat. Certes, la région 
Languedoc-Roussillon n'est pas absente des politiques communautaires mais s'en saisit là aussi de façon différente, selon d'autres enjeux, non identitaires. Au niveau de ce que certains peinent à appeler "Catalogne nord", la divergence au niveau des objectifs, le manque de coordination qui peut relever d'un choix, ont limité le potentiel dont pourrait bénéficier le département. De ce fait, s'y confirme une sorte de doublon entre un réseau de coopération interurbaine initiée par J.P Alduy et une coopération "transfrontalière" défendue au niveau des pays frontaliers. En Catalogne, le gouvernement régional reste le principal maître d'œuvre de ses politiques européennes à travers des comarques, établissements publics voire acteurs privés coordonnés selon des attentes pragmatiques en termes d'équipements (Trains à Grande Vitesse ou Tren Alta Valocitat, ports) et de projection politique extérieure.

Contrairement à la configuration dans les Pyrénées-Orientales, le gouvernement catalan est un acteur fort avec des compétences qui lui permettent de coordonner ses collectivités, villes et autres, à travers une continuité, malgré les changements de personnalités politiques, ou encore les quelques conflits internes, et une solide culture de portage de projets européens acquise depuis les années 1980. Au niveau de nos espaces d'études choisis, notamment pour les parties françaises, cette affirmation d'un responsable d'un établissement public en Cerdagne que nous avions interrogé illustre très bien les conflits entre acteurs et les types de coordination politique, à l'œuvre : il y a trop de structures: PNR, Communautés de Communes, Terres Romanes, en plus des inter-consulaires (commerce et métiers). Tout ça est pourtant géré par Bourquin [Président de la Région]. Donc, tout le décisionnel est socialiste et les deux locaux (maires de Saillagouse et de Fontrabiouse) ne sont pas dans la mouvance du département et de la Région. Pire, la communauté de communes capcirente est socialisante, seulement pour 3000 habitants. Donc, c'est là qu'il faut relativiser cet espace. En Espagne, c'est beaucoup plus facile avec un rayon d'action beaucoup plus large avec les comarques. Il faut des leaderships.

\section{Conclusion : des frontières modèles ou des modèles de territoires transfrontaliers?}

Les élus locaux savent aussi se saisir de la coopération pour mettre en œuvre de projets relevant du tourisme: la restauration du site de Paulilles par le Conseil Général des Pyrénées-Orientales est un exemple dans le département (réaménagement et tourisme). A l'échelle transfrontalière, il y a le projet de voie verte Enllaç, porté par quelques collectivités dans le Vallespir et l'Emporda. A posteriori, il n'y a pas de thèmes modèles de coopération, encore moins de projets modèles. Par conséquent, l'on peut éventuellement parler de frontière modèle selon la gouvernance, ou selon des dynamiques propres ou générales (dans ce cas, l'on pourrait parler de type de frontière) ou de structures exemplaires. Malgré le projet de l'Eurodistrict Catalan Transfrontalier et son échec avorté, selon Henri Sicre, ancien député, la coopération de proximité ne peut pas être la même sur tout le territoire; la coopération reste une démarche volontariste qui doit s'adapter aux réalités topographiques. Sur la Cerdagne, nous pouvons relever, comme l'ont déjà souligné Séchet et Keerle, que c'est moins sous l'angle de l'emploi et des apports économiques que sous celui des constructions territoriales qu'est posée la question des enjeux de la constitution d'un espace de santé transfrontalier. 
Tableau 1. Synthèse.

\begin{tabular}{|c|c|c|c|c|}
\hline \multirow[b]{2}{*}{ Projets choisis } & \multicolumn{2}{|c|}{ Zone d'étude I } & \multicolumn{2}{|l|}{ Zone d'étude II } \\
\hline & PRINCALB & MIRO & HTC (Hôpital) & MTC (Abattoir) \\
\hline Thèmes de coopération & \multicolumn{2}{|c|}{$\begin{array}{l}\text { Environnement / } \\
\text { Tourisme }\end{array}$} & \multicolumn{2}{|c|}{ Santé (en lien avec tourisme) } \\
\hline $\begin{array}{l}\text { Principales structures de gouvernance } \\
\text { transfrontalière }\end{array}$ & \multicolumn{2}{|c|}{$\begin{array}{l}\text { - } \quad \text { Albera } \quad \text { Viva } \\
\text { (Associations) }\end{array}$} & \multicolumn{2}{|c|}{$\begin{array}{l}\text { - GECT Pyrénées Cerdagne } \\
\text { - GECT Hôpital }\end{array}$} \\
\hline Nombre de structures & \multicolumn{2}{|c|}{3 repertoriées } & \multicolumn{2}{|l|}{35 répertoriées } \\
\hline Type de frontière & \multicolumn{2}{|c|}{ Montagne et Littoral } & \multicolumn{2}{|c|}{ Plaine semi urbanisée } \\
\hline Typologie de proximité & \multicolumn{2}{|c|}{ Géographique } & \multicolumn{2}{|l|}{$\begin{array}{l}\text { Géographique, } \\
\text { Institutionnel }\end{array}$} \\
\hline
\end{tabular}

Les deux espaces choisis constituent des territoires intégrés, un territoire fonctionnel pour la Cerdagne, et un dont la gouvernance de proximité est en cours de structuration. Au niveau des Albères, du Vallespir et de l'Empordà, nous tenons toutefois à nuancer cette structuration territoriale : la géographie est la même ; il existe un pôle de chalandise au niveau du Perthus, un pôle d'échange ferroviaire au niveau de Cerbère / Port-Bou mais ces derniers restent encore utilitaires et disparates, et ne répondent pas à la même logique d'aménagement. De ce fait, même en matière d'urbanisme, il n'y a pas de coordinations malgré le potentiel d'une planification intégrée. Le défi de l'Eurodistrict pourrait être la coordination du bassin de vie cerdan ou du moins ce bassin sanitaire avec les formations et la recherche au niveau de Perpignan, en lien avec les universités catalanes et les problématiques des espaces frontaliers concernés; ou encore, d'une planification concertée des projets de parcs éoliens au niveau de l'Empordà et des Albères, susceptible là aussi de faire face à une mobilisation locale. Il ressort de l'étude que le plateau cerdan disposant de plus de structures transfrontalières est plus articulé de part et d'autre. En guise de structure exemplaire, le GECT Hôpital de Cerdagne peut être cité. Ce dernier institué le 26 avril 2010, est prévu pour 10 ans et réalise un service public (la gestion de l'assistance médicale) sur un périmètre transfrontalier comprenant 53 communes françaises et espagnoles. En remplaçant la fondation de droit privé catalane qui était chargée de la construction de l'Hôpital de Cerdagne, ce GECT illustre la solution donnée à un vide juridique de la part des instances communautaires et la coordination au niveau local pour la gestion d'un équipement hospitalier et la prestation de services dans d'autres établissements (dont par exemple la Maison Médicale de santé Joseph Sauvy à Err) pour des populations résidant dans deux entités étatiques. 


\section{BIBLIOGRAPHIE}

ARMANGUE J. (2010), Una Idea De L'empordà Dins L'espai Català Transfronterer, Figuères, EDXS Edicions, $135 \mathrm{p}$.

BRENNER N. (2004), New states spaces, Urban Governance and the Rescaling of Statehood, Oxford, Oxford University Press.

CASTEX-EY J.F. (2012), Le défi de la diversité territoriale dans l'Espace Catalan Transfrontalier, article mis en ligne sur le site du Think Tank "OpenCat", http://www.opencat.info/article/fr/1327/ledefi-de-la-diversite-territoriale-dans-lespace-catalan-transfrontalier.

FOURNY M.-C. (2005), "La frontière, objet d'intermédiation territoriale," Peuples et Monde, Globalisation et peuples aux frontières de l'Europe, $10 \mathrm{p}$.

FOURNY M.-C., CRIVELLI R. (2003), “Cette montagne que l'on partage. L'identification de la montagne et de la frontière dans les coopérations transfrontalières de régions alpines", Revue de Géographie Alpine, $\mathrm{n}^{\circ}$ 3, pp. 57-70.

GIBAND D. (Coord.) (2009), “Catalogne - Catalognes”, Revue Sud Ouest Européen, 28, Toulouse, Presses Universitaires du Mirail, $119 \mathrm{p}$.

GIBAND D. (2011), Les villes de la diversité, Territoires du vivre ensemble, Paris, Anthropos, Economica, $150 \mathrm{p}$.

LE BART C. et LEFEBVRE R. (dir.) (2005), La proximité en politique : Usages, rhétoriques, pratiques, Rennes, Presses universitaires de Rennes, $305 \mathrm{p}$.

LE GALES P. (1995), “Du gouvernement des villes à la gouvernance urbaine”. Revue française de science politique, 1,. pp. 57-95.

MANCEBOT F. (1999), La Cerdagne et ses frontières : conflits et identités transfrontalières, Perpignan, Editions Llibres del Trabucaïre, $211 \mathrm{p}$.

MAURY C. (2008), "Faut-il nécessairement "s'aimer" pour coopérer entre Européens ? Deux exemples transfrontaliers", Politique européenne, 3 26, pp. 75-95.

MOULLE F. \& DUHAMEL S. (Coord.) (2010), Frontières et santé. Genèse et maillage des réseaux transfrontaliers, Paris, L'Harmattan, 286 p.

OLIVAREZ GONZALEZ X. (2009), La construcció metageogràfica de l'Arc Mediterrani, Thèse doctorale, Département de Géographie, Université Autonome de Barcelone, Volume II, 233 p.

PAYROT M. (2010), “La protection transfrontalière du massif naturel de l'Albera contre les incendies", RECERC, Collection Europe et frontières, 5, $173 \mathrm{p}$.

PUIGNEAU A. (2000), Projet de création d'un parc naturel régional transfrontalier sur les Albères, Mémoire de Maîtrise d'Aménagement du Territoire.

SECHET R.et KEERLE R. (2010), “Le projet d'Hôpital commun transfrontalier de Cerdagne : des difficultés de la coopération transfrontalière en matière de santé dans un contexte pourtant favorable", Annales de Géographie, 675, pp. 536-559

TORRE A. (2009), "Retour sur la notion de proximité géographique”. Géographie, économie, société, 11, pp. 63-75. 


\section{NOTES}

1. D'après les chiffres de la Mission Opérationnelle Transfrontalière, seulement $1 \%$ des actifs (environ 700 personnes) des Pyrénées-Orientales rejoignent quotidiennement le côté sud de la frontière pour leur emploi et il s'agit majoritairement de personnes résidant en Cerdagne.

\section{RÉSUMÉS}

Dans le cadre de sa politique régionale, l'Union Européenne a récemment appelé à une coopération entre acteurs de proximité par son règlement de 2006, parallèlement à la création du GECT (Groupement Européen de Coopération Territoriale). L'objectif de cet article est de construire un modèle de représentation et d'évaluation de la gouvernance d'une frontière, en l'occurrence la partie orientale de la frontière transpyrénéenne. Il s'agit aussi d'analyser la problématique du voisinage ou de la contigüité entre espaces frontaliers avec la rhétorique de la coopération de proximité : comment se structurent ou non ces dynamiques? Cet article s'articule en trois principaux points : une contextualisation et des éléments méthodologiques, complétés par une présentation du périmètre d'étude, ensuite les résultats obtenus sur les types de frontières et les axes de coopération.

In the framework of its regional policy, the European Union has recently encouraged cooperation between local actors with the Regulation of 2006 on the creation of the EGTCs (European Groupings of Territorial Cooperation). The objective of this paper is to build a model of representation of a boundary (in this case the border crossing the Eastern Pyrenees) and of evaluation of its governance. It is also aims to examine the issue of neighbourhood or contiguity between border areas with the rhetoric of proximity cooperation: how are these processes structured (or not). The paper is divided into three main parts: contextualization and methodological elements, presentation of the study area, results on the types of borders and areas of cooperation.

\section{INDEX}

Mots-clés : coopération transfrontalière, proximité, gouvernance, Cerdagne, Capcir, Vallespir, Albères, Catalogne

Keywords : cross-border cooperation, proximity, governance, Catalonia

\section{AUTEUR}

\section{ADENANE DJIGO}

UMR ART-DEV 5281, Acteurs, Réseaux et Territoires dans le développement, Université de Perpignan, Faculté LSH, 52 Avenue Paul Alduy, 66860, Perpignan, Cedex, France, adenane.djigo@univ-perp.fr 